\title{
Double Domain Swapping in Human $\gamma C$ and $\gamma D$ Crystallin Drives Early Stages of Aggregation
}

Published as part of The Journal of Physical Chemistry virtual special issue "Dave Thirumalai Festschrift".

\author{
Balaka Mondal, Jayashree Nagesh, and Govardhan Reddy*
}

Cite This: J. Phys. Chem. B 2021, 125, 1705-1715

Read Online

ACCESS

Wll Metrics \& More

Article Recommendations

Supporting Information

ABSTRACT: Human $\gamma \mathrm{D}(\mathrm{H} \gamma \mathrm{D})$ and $\gamma \mathrm{C}(\mathrm{H} \gamma \mathrm{C})$ are two-domain crystallin (Crys) proteins expressed in the nucleus of the eye lens. Structural perturbations in the protein often trigger aggregation, which eventually leads to cataract. To decipher the underlying molecular mechanism, it is important to characterize the partially unfolded conformations, which are aggregation-prone. Using a coarse grained protein model and molecular dynamics simulations, we studied the role of on-pathway folding intermediates in the early stages of aggregation. The multidimensional free energy surface revealed at least three different folding pathways with the population of partially structured intermediates. The two dominant pathways confirm sequential folding of the $\mathrm{N}$-terminal [Ntd] and

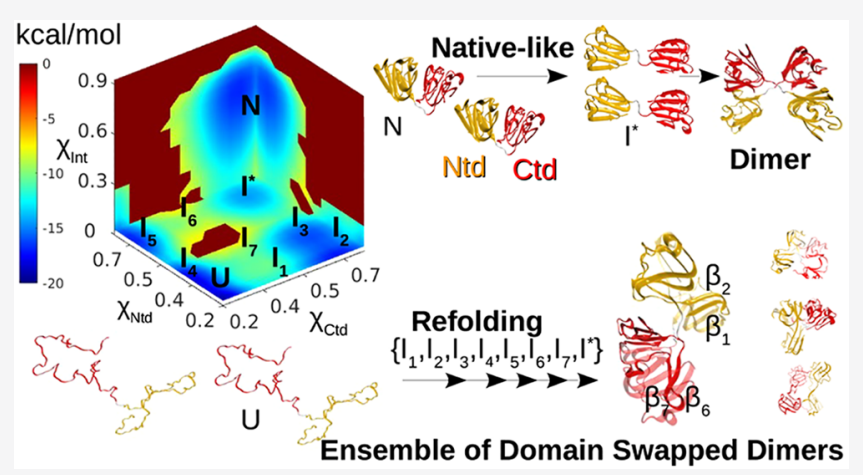
the C-terminal domains [Ctd], while the third, least favored, pathway involves intermediates where both the domains are partially folded. A native-like intermediate $\left(I^{*}\right)$, featuring the folded domains and disrupted interdomain contacts, gets populated in all three pathways. $I^{*}$ forms domain swapped dimers by swapping the entire Ntds and Ctds with other monomers. Population of such oligomers can explain the increased resistance to unfolding resulting in hysteresis observed in the folding experiments of $\mathrm{H} \gamma \mathrm{D}$ Crys. An ensemble of double domain swapped dimers are also formed during refolding, where intermediates consisting of partially folded Ntds and Ctds swap secondary structures with other monomers. The double domain swapping model presented in our study provides structural insights into the early events of aggregation in Crys proteins and identifies the key secondary structural swapping elements, where introducing mutations will aid in regulating the overall aggregation propensity.

\section{INTRODUCTION}

Lens opacity or cataract $^{1}$ is a protein aggregation disorder associated with misfolding and subsequent aggregation of eye lens crystallin (Crys) proteins, resulting in partial or complete loss of vision. Detailed structural characterization of the misfolded states and molecular mechanism of cataract formation have remained elusive to date. In humans, the surgically removed cataractous lenses contain high molecular weight aggregates of $\alpha, \beta$, and $\gamma$ Crys, the three main classes of Crys found in vertebrates. ${ }^{2,3} \alpha$ Crys primarily acts as molecular chaperones, ${ }^{4}$ while $\beta$ and $\gamma$ Crys are responsible for optical functions of the lens. ${ }^{5,6}$

Monomeric $\gamma$ Crys are the simplest members of the Crys family. The three most abundant $\gamma$ Crys proteins in humans are $\mathrm{H} \gamma \mathrm{C}, \mathrm{H} \gamma \mathrm{D}$, and $\mathrm{H} \gamma \mathrm{S}$ Crys, which are present in the central region of the lens. $\mathrm{H} \gamma \mathrm{C}$ and $\mathrm{H} \gamma \mathrm{D}$ Crys are expressed in the lens nucleus, ${ }^{7}$ which is the oldest region of the lens with no protein turnover. Since these proteins are only synthesized once, the native state of these proteins should be stable and functional for the human lifetime. Any misfolding significantly perturbs the equilibrium concentrations of $\mathrm{H} \gamma \mathrm{C}$ and $\mathrm{H} \gamma \mathrm{D}$ Crys in the lens.

$\mathrm{H} \gamma \mathrm{C}$ and $\mathrm{H} \gamma \mathrm{D}$ Crys are both 173 residue long proteins with $\approx 71 \%$ sequence similarity. The native structure of $\gamma$ Crys is topologically complex with a $\mathrm{N}$-terminal domain $(\mathrm{Ntd})$ and a C-terminal domain (Ctd), which are highly homologous. Each domain consists of two intercalated greek-key motifs, which are supersecondary structures formed by four antiparallel $\beta$ strands (Figures $1 \mathrm{~A}$ and $2 \mathrm{~A}$ ). Intercalated greek key motifs termed $M_{1}$ and $M_{2}$ constitute the $\mathrm{Ntd}$, while $M_{3}$ and $M_{4}$ constitute the $\mathrm{Ctd}^{8}$ (Figure 1B). A short peptide linker connects the two domains, which form a highly conserved hydrophobic interface necessary for the long-term stability of the proteins. ${ }^{9}$ The

Received: August 28, 2020

Revised: January 27, 2021

Published: February 10, 2021

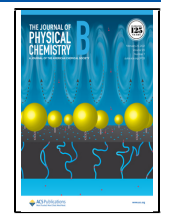




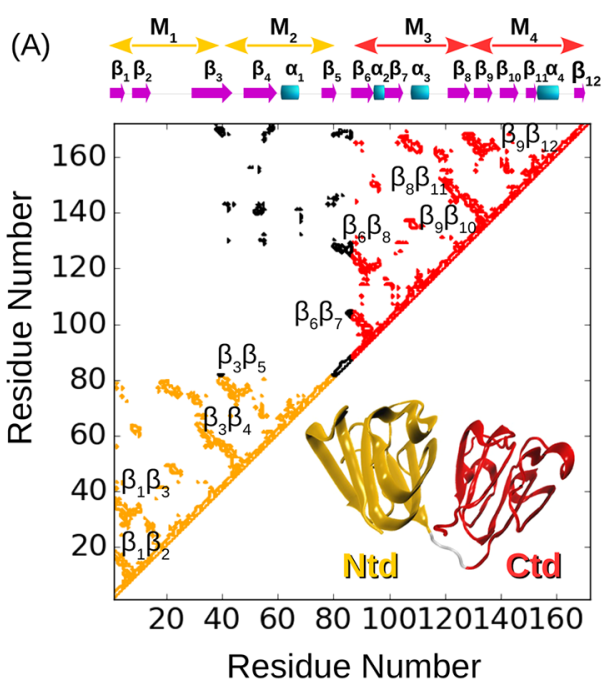

(D)

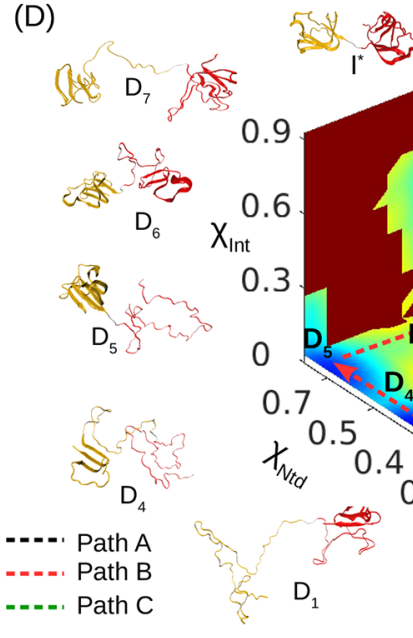

(B)

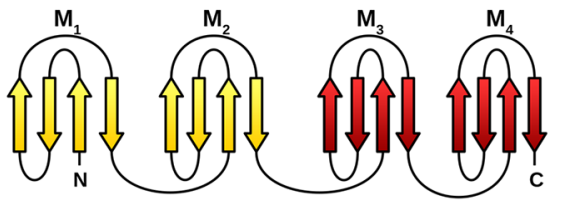

(C)

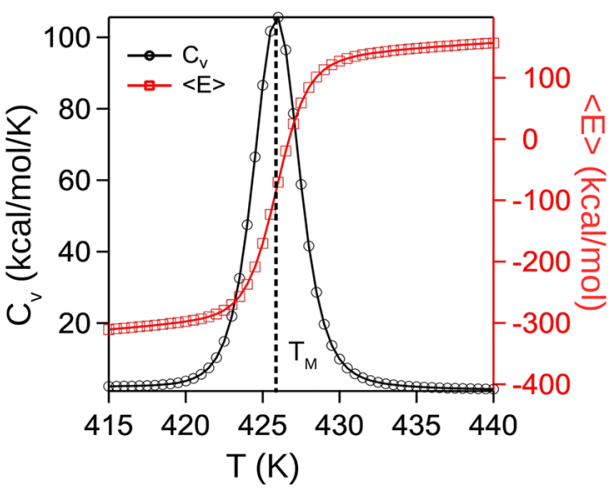

(E)

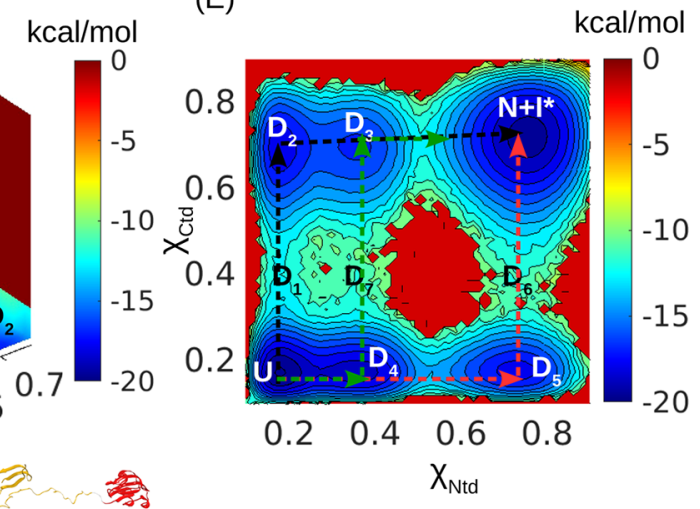

Figure 1. (A) Contact map of $\mathrm{H} \gamma \mathrm{D}$ Crys ${ }^{46}$ (PDB ID: $1 \mathrm{HK} 0$ ). $\mathrm{H} \gamma \mathrm{D}$ Crys consists of two domains called N-terminal domain (Ntd, shown in orange) and C-terminal domain (Ctd, shown in red) connected by a short peptide linker. Each domain is further composed of two intercalated greek key motifs. Greek key motifs constituting the Ntd are termed as $M_{1}$ (residues Gly1 to Ser39) and $M_{2}$ (residues Gly40 to Pro82), while motifs constituting the Ctd are termed as $M_{3}$ (residues His88 to Glu128) and $M_{4}$ (residues Gly129 to Ile171). The intradomain contacts are colored orange ( $\mathrm{Ntd})$ and red $(\mathrm{Ctd})$, while interdomain contacts are colored black. (B) A splay diagram of four ideal interconnected greek key motifs. Four antiparallel $\beta$-strands constitute one greek key motif where strands $\beta_{1}$ and $\beta_{3}$ are in contact. (C) Temperature-dependent variation in specific heat capacity $\left(C_{v}\right)$ and average potential energy $(\langle E\rangle)$ indicate a two-state folding transition in H $\gamma \mathrm{D}$ Crys. (D) FES, $F\left(\chi_{\mathrm{Ntd}}, \chi_{\mathrm{Ctd}}, \chi_{\mathrm{Int}}\right)$ projected onto a fraction of native contacts in $\mathrm{Ntd}\left(\chi_{\mathrm{Ntd}}\right)$, in $\mathrm{Ctd}\left(\chi_{\mathrm{Ctd}}\right)$, and between the two domains $\left(\chi_{\mathrm{Int}}\right)$ at $T_{\mathrm{M}}=426 \mathrm{~K}$. Three pathways connect the native state $(N)$ and the unfolded state $(U)$ through distinct intermediate states. Representative structures of the intermediates are shown. $(\mathrm{E}) F\left(\chi_{\mathrm{Ntd}}, \chi_{\mathrm{Ctd}}\right)$ projected onto $\chi_{\mathrm{Ntd}}$ and $\chi_{\mathrm{Ctd}}$. All the intermediate states are visible except for $I^{*}$ (Ctd and $\mathrm{Ntd}$ domains are folded with disrupted interdomain contacts), which is indistinguishable from the $N$ state.

topology of the native state of $\gamma$ Crys leads to a complex folding behavior, as observed in experiments ${ }^{9-12}$ and computer simulations. $^{13-15}$

Due to their implications in cataract, biophysical properties of $\gamma$ Crys have been studied extensively using both experimental and computational techniques. Studying folding in multidomain proteins is challenging since the domaindomain interactions add to the complexity, as they can influence folding in addition to the intradomain interactions. ${ }^{16-19}$ Denaturant-induced unfolding experiments ${ }^{10,11,20,21}$ demonstrated a three-state unfolding transition in $\gamma$ Crys, indicating the presence of a partially structured intermediate state. This intermediate state is structurally characterized for $\mathrm{H} \gamma \mathrm{D}$ Crys, which consists of a folded Ctd and an unfolded $\mathrm{Ntd},{ }^{9,22}$ in agreement with the simulations. ${ }^{13}$
Hysteresis is another interesting property observed in the denaturant-induced equilibrium unfolding/refolding experiments. ${ }^{10,23,24}$ Native states of $\mathrm{H} \gamma \mathrm{D}$ Crys are observed to be stable at higher denaturant concentrations where unfolded states did not refold. Increased temperature or equilibration time led to diminished hysteresis, causing a shift in the unfolding transition only, indicating a high kinetic barrier to unfolding as a possible reason for hysteresis. Further, increased stability of the interdomain interface led to enhanced hysteresis, which is absent in the truncated domains. ${ }^{11}$ Thus, the interdomain interactions are attributed to the high kinetic barriers in the early stages of unfolding. ${ }^{24,25}$ However, the origin of hysteresis is yet to be explored.

Experiments $^{10,26,27}$ further revealed a competitive protein aggregation pathway during the refolding of $\mathrm{H} \gamma \mathrm{D}$ and $\mathrm{H} \gamma \mathrm{C}$ Crys at $[\mathrm{GdmCl}]<1 \mathrm{M}$ due to the population of partially 


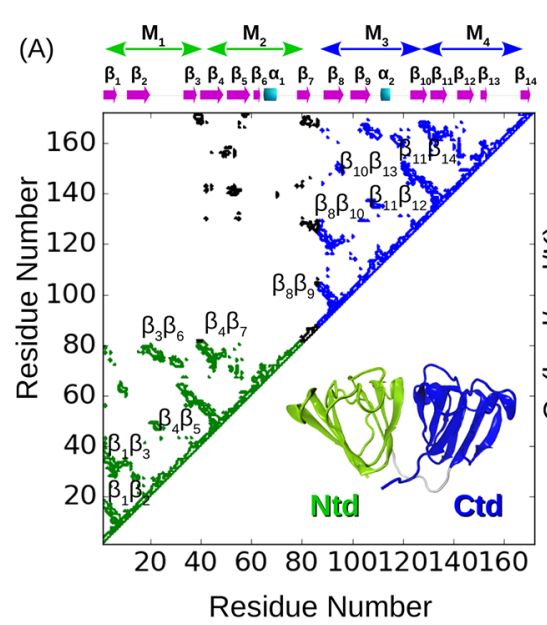

(B)

(C)
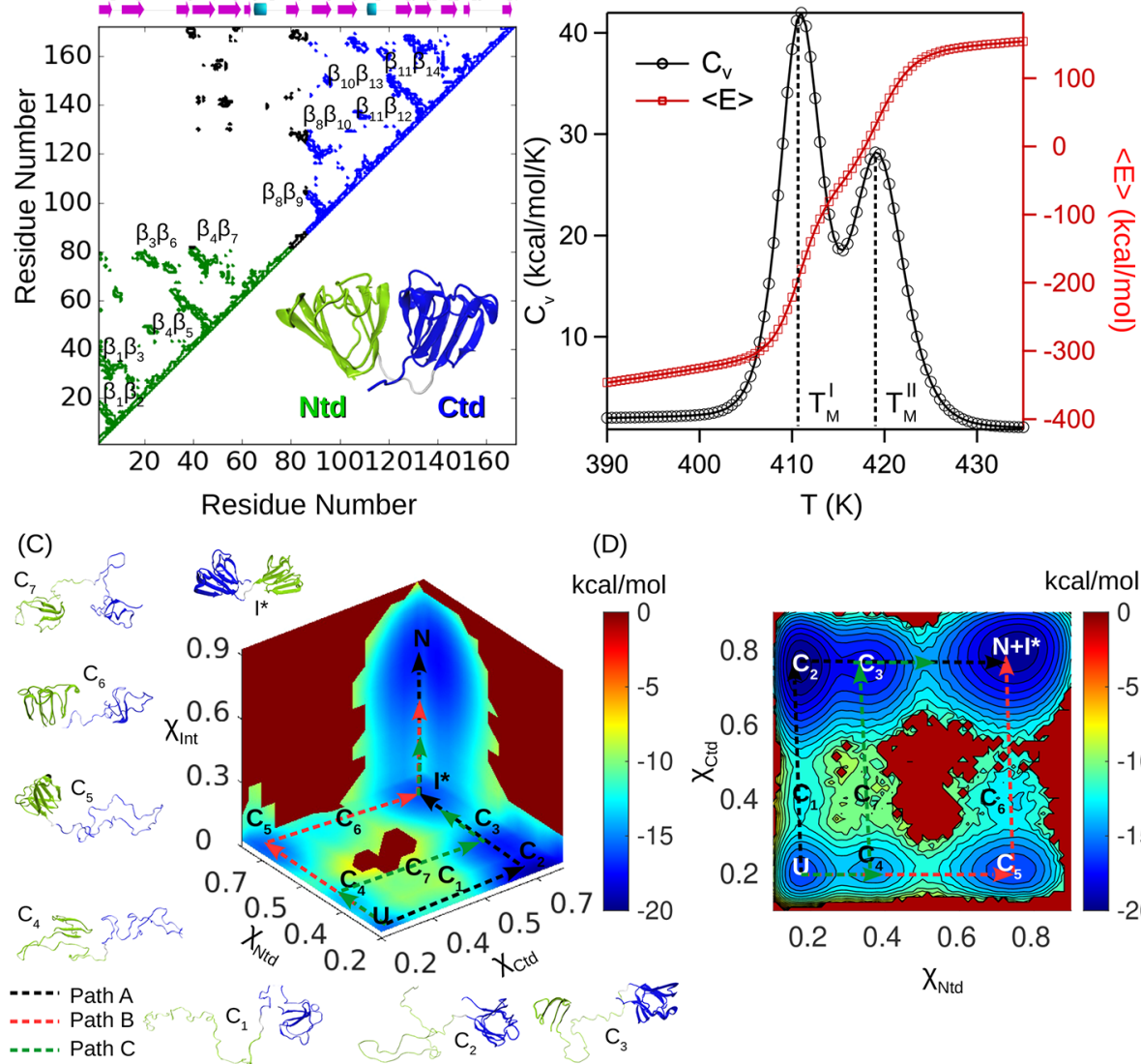

Residue Number

(D)
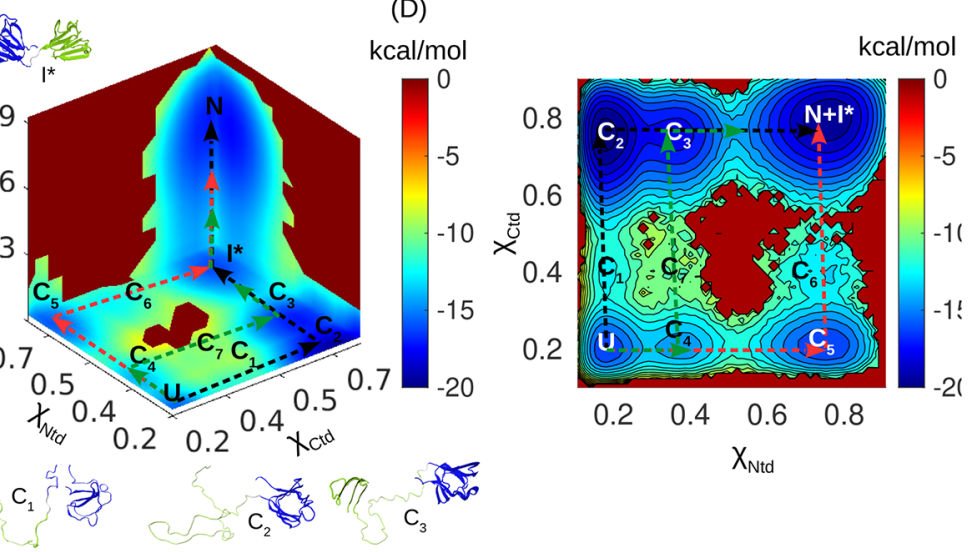

Figure 2. (A) Contact map of $\mathrm{H} \gamma \mathrm{C}$ Crys ${ }^{46}$ (PDB ID: 2NBR). ${ }^{45} \mathrm{H} \gamma \mathrm{C}$ Crys consists of two domains called N-terminal domain (Ntd, shown in green) and C-terminal domain (Ctd, shown in blue) connected by a short peptide linker. Each domain is further composed of two intercalated greek key motifs. Greek key motifs constituting the Ntd are termed as $M_{1}$ (residues Gly1 to Ser39) and $M_{2}$ (residues Gly40 to Pro82), while motifs constituting the Ctd are termed as $M_{3}$ (residues His87 to Glu127) and $M_{4}$ (residues Gly128 to Val170). The intradomain contacts are colored green $(\mathrm{Ntd})$ and blue $(\mathrm{Ctd})$, while interdomain contacts are colored black. (B) Temperature-dependent variations in $C_{v}$ and $\langle E\rangle$ indicate a three state folding transition in $\mathrm{H} \gamma \mathrm{C}$ Crys. (C) $F\left(\chi_{\mathrm{Ntd}}, \chi_{\mathrm{Ctd}}, \chi_{\mathrm{Int}}\right)$ projected onto $\chi_{\mathrm{Ntd}} \chi_{\mathrm{Ctd}}$ and $\chi_{\mathrm{Int}}$ at $T_{\mathrm{M}}^{\mathrm{I}}=410 \mathrm{~K}$. Three pathways connect the $N$ state and $U$ state through distinct intermediate states. Representative structures of the intermediates are shown. (D) $F\left(\chi_{\mathrm{Ntd}}, \chi_{\mathrm{Ctd}}\right)$ projected onto $\chi_{\mathrm{Ntd}}$ and $\chi_{\mathrm{Ctd}}$ at $T_{\mathrm{M}}^{\mathrm{I}}$. All the intermediate states are visible except for $I^{*}$ (Ctd and $\mathrm{Ntd}$ domains are folded with disrupted inter domain contacts), which is indistinguishable from the $N$ state.

folded intermediates. ${ }^{10}$ Domain swapping ${ }^{28-33}$ plays an important role in the aggregation of globular proteins such as prion proteins, ${ }^{34}$ cystatin $\mathrm{C},{ }^{35,36} \mathrm{c}$-Src SH $3,{ }^{37} \beta 2$-microglobulin, ${ }^{38}$ and so on, where it is believed to drive the early stages of aggregation. In proteins such as RNase A, which can swap secondary structures from both $\mathrm{Ntd}$ and Ctd, double domain swapping is observed, leading to a variety of differently assembled oligomeric states. ${ }^{39-42}$ To elucidate the structure of aggregation prone intermediates and the underlying aggregation mechanism in Crys, several studies have investigated the dimer formation in $\mathrm{H} \gamma \mathrm{D}$ Crys, which is the simplest oligomer. $^{12,14,43}$ A transient intermediate state with an extruded $\mathrm{N}$ terminal $\beta$ hairpin loop is identified in single molecule atomic force microscopy (AFM) experiments ${ }^{12}$ and computer simulations, ${ }^{43}$ which undergoes dimerization, yielding different dimer structures in experiments and simulations. Although the molecular events leading to aggregation are not fully understood, domain swapping is considered to be the most probable aggregation mechanism $^{12,14,43}$ in $\mathrm{H} \gamma \mathrm{D}$ Crys.

All-atom simulations predicted domain-swapped structures, where the $\mathrm{Ntd}$ of one monomer interacts with the three $\beta$ strands from $M_{4}$ of $\mathrm{Ctd}$ from another monomer, ${ }^{14}$ and $\beta_{1}$ from the extruded $\beta$ hairpin loop in Ntd forms antiparallel hydrogen bonds with the second $\beta$ strand from $M_{4}$ of $\mathrm{Ctd}^{43}$ These structures remain to be validated experimentally. AFM experiments on $\mathrm{H} \gamma \mathrm{D}$ Crys indicated a different domain swapped structure with $\mathrm{Ntd} \beta$ hairpin loops swapped between adjacent monomers. ${ }^{12}$ Experiments also suggest that the aggregation-prone intermediate is not structurally similar to the unfolding intermediate and consists of a partially folded Ctd. $^{44}$

To probe the reasons behind the observed hysteresis during the folding of $\gamma$ Crys and to gain insight into the aggregationprone misfolded intermediates, we studied the folding thermodynamics of $\mathrm{H} \gamma \mathrm{C}$ (protein data bank (PDB) ID: $2 \mathrm{NBR})^{45}$ and $\mathrm{H} \gamma \mathrm{D}$ (PDB ID: $\left.1 \mathrm{HK} 0\right)^{46}$ Crys using molecular dynamics simulations and coarse grained self-organized polymer side-chain (SOP-SC) protein model. ${ }^{47,48}$ The folding free energy surface (FES) constructed from low friction Langevin dynamics simulations shows that $\mathrm{H} \gamma \mathrm{C}$ and $\mathrm{H} \gamma \mathrm{D}$ Crys exhibit kinetic partitioning mechanism and folds through at least three distinct pathways populating different intermediate states. One of the intermediates is an early unfolding 
intermediate with both domains folded and an exposed interface. Through the population of this intermediate we show that the native proteins form a domain-swapped dimer, which is a more stable entity with four folded domains and two interfaces. The population of this dimer structure during the early stages of unfolding can lead to hysteresis by resisting unfolding and requiring relatively higher denaturant concentrations to unfold. Experiments report similar domain-swapped dimer structures for human $\beta \mathrm{B} 2 \mathrm{Crys}^{49-51}$ (PDB ID: 2BB2) and mouse $\gamma \mathrm{S}$ Crys (PDB ID: 6MYG, 6MYH).

Experiments ${ }^{10}$ further show that $\gamma$ Crys exhibits significant aggregation while refolding at lower denaturant concentrations. To gain insight into this in vivo aggregation, we carried out dimerization simulations of the unfolded proteins. During dimerization, secondary structural elements from partially folded $\mathrm{Ntd}$ and Ctd of the proteins took part in domainswapping, leading to an ensemble of double domain swapped structures. The primary swapping units in $\mathrm{H} \gamma \mathrm{C}$ and $\mathrm{H} \gamma \mathrm{D}$ Crys were found to be very similar, which can be attributed to their high sequence similarity $(\approx 71 \%)$. Both in $\mathrm{H} \gamma \mathrm{D}$ and $\mathrm{H} \gamma \mathrm{C}$ Crys, $\mathrm{Ntds}$ undergo aggregation by swapping the $\beta_{1} \beta_{2}$ hairpin loop, while Ctds swap the first hairpin loop in motif $M_{3}\left(\beta_{6} \beta_{7}\right.$ in $\mathrm{H} \gamma \mathrm{D}$ Crys, $\beta_{8} \beta_{9}$ in $\mathrm{H} \gamma \mathrm{C}$ Crys). Other less common swapping units identified from the dimer structures include strands $\beta_{1}$, $\beta_{3} \beta_{4}$, motif $M_{1}$ in the $\mathrm{Ntd}$ of $\mathrm{H} \gamma \mathrm{D}$ Crys (strands $\beta_{1}, \beta_{7}$ and motif $M_{1}$ in the $\mathrm{Ntd}$ of $\mathrm{H} \gamma \mathrm{C}$ Crys), and strands $\beta_{6}, \beta_{12}$ in the Ctd of $\mathrm{H} \gamma \mathrm{D}$ Crys $\left(\beta_{8}, \beta_{14}\right.$ in the Ctd of $\mathrm{H} \gamma \mathrm{C}$ Crys). AFM experiments $^{12}$ on $\mathrm{H} \gamma \mathrm{D}$ Crys also predicted a domain swapped dimer structure where the $\mathrm{N}$-terminal $\beta$ hairpin loops get swapped between the monomers. Interestingly, a large number of cataract-associated mutations are found in the $\beta_{1} \beta_{2}$ loop region in $\mathrm{H} \gamma \mathrm{D}$ Crys. ${ }^{24,52-55}$ Such a double domain swapping mechanism is feasible in an end to end fashion ${ }^{56}$ among specific partially structured intermediates, giving rise to long chains of interconnected oligomers, which might act as aggregation precursors in cataractous lenses.

\section{MATERIALS AND METHODS}

Molecular dynamics simulations are performed using coarsegrained self-organized polymer side-chain (SOP-SC) $)^{47,48}$ description of the human $\gamma$ Crys proteins. In SOP-SC model, each amino acid is represented by two beads, one placed at the center of the $\mathrm{C}_{\alpha}$ atom, while another is placed at the center of mass of the side chain. Coarse-grained structures of the proteins are built from their PDB structures with PDB IDs $2 \mathrm{NBR}^{45}\left(\mathrm{H} \gamma \mathrm{C}\right.$ Crys) and $1 \mathrm{HKO}^{46}(\mathrm{H} \gamma \mathrm{D}$ Crys $)$. The Hamiltonian of a protein conformation in the SOP-SC model is given by

$$
E_{\mathrm{CG}}=E_{\mathrm{B}}+E_{\mathrm{NB}}^{\mathrm{N}}+E_{\mathrm{NB}}^{\mathrm{NN}}+E_{\mathrm{el}}
$$

where $E_{\mathrm{B}}$ is the energy due to bonded interaction present between the covalently bonded beads, $E_{\mathrm{NB}}^{\mathrm{N}}$ and $E_{\mathrm{NB}}^{\mathrm{NN}}$ are the energy due to nonbonded native and non-native interactions, respectively, and $E_{\mathrm{el}}$ is the energy due to electrostatic interactions present between the charged residues in the protein. Native interactions exist between two beads, if they are separated by at least three bonds and are within a cutoff distance $\left(R_{\mathrm{c}}=8 \AA\right)$ in the SOP-SC model of the PDB structure, while any other noncovalent interactions are considered as non-native. Bonded interactions are modeled using the finite extensible nonlinear elastic (FENE) potential, while native (non-native) interactions are modeled using a
Lennard-Jones-like (repulsive term of Lennard-Jones) potential. Electrostatic interactions are implemented using a screened Coulomb potential. Interaction energies between side-chain beads are obtained from the Betancourt-Thirumalai statistical potential. ${ }^{57}$ Symmetric Go potential ${ }^{32,58}$ (Figure $S 1$ ) is used to model the interactions between two protein monomers, which is discussed in detail in the Supporting Information (SI). Langevin dynamics simulations are used to compute equilibrium thermodynamic properties. The weighted histogram analysis method (WHAM) is used to construct the three-dimensional free energy landscape $F\left(\chi_{\mathrm{Ntd}}, \chi_{\mathrm{Ctd}}, \chi_{\mathrm{Int}}\right)$ from the Langevin dynamics simulations performed at various temperatures. Brownian dynamics ${ }^{59}$ simulations are performed to study dimerization kinetics of the proteins. A detailed description of the protein models, simulation methodology, and parameters is provided in the SI.

\section{RESULTS AND DISCUSSION}

Folding Thermodynamics of $\mathrm{H} \gamma \mathrm{C}$ and $\mathrm{H} \gamma \mathrm{D}$ Crys. Folding of $\gamma$ Crys proteins have been studied using different experimental methods, including fluorescence spectroscopy, circular dichroism, AFM, and so on. ${ }^{10-12,22,44}$ However, a detailed characterization of the transient intermediates populated in the folding pathways still remains a challenge. We performed low friction Langevin dynamics simulations to study the folding of $\mathrm{H} \gamma \mathrm{C}$ and $\mathrm{H} \gamma \mathrm{D}$ Crys using a coarse-grained SOP-SC protein model. ${ }^{47,48}$ A detailed description of the SOPSC protein model and simulation parameters are in the SI. The simulations are performed at temperatures ranging from $T=$ $300-500 \mathrm{~K}$ and we computed various thermodynamic properties associated with the folding.

Temperature-dependent variation in specific heat capacity $\left(C_{v}\right)$ and average potential energy $(\langle E\rangle)$ indicate a cooperative two-state folding for $\mathrm{H} \gamma \mathrm{D}$ Crys with the melting temperature, $T_{\mathrm{M}} \approx 426 \mathrm{~K}$ (Figure $1 \mathrm{C}$ ). Whereas a three-state folding with the population of an intermediate is observed for $\mathrm{H} \gamma \mathrm{C}$ Crys with two peaks in the $C_{v}$ plot at $T_{\mathrm{M}}^{\mathrm{I}} \approx 410 \mathrm{~K}$ and $T_{\mathrm{M}}^{\mathrm{II}} \approx 420 \mathrm{~K}$ (Figure 2B). Experimentally ${ }^{26,60}$ measured $T_{M}$ values for $\mathrm{H} \gamma \mathrm{C}$ and $\mathrm{H} \gamma \mathrm{D}$ Crys are 352 and $357 \mathrm{~K}$, respectively. The deviation in the $T_{M}$ obtained from experiments and simulations is due to the coarse-grained level description used to model the proteins, which cannot accurately mimic the experimental conditions. Experimental studies on $\mathrm{H} \gamma \mathrm{D}$ and $\mathrm{H} \gamma \mathrm{C}$ Crys further indicated a three-state equilibrium unfolding with the population of an intermediate state. ${ }^{10}$ Although the $C_{v}$ plot associated with $\mathrm{H} \gamma \mathrm{C}$ Crys indicates a three-state folding/unfolding transition with an intermediate, only a two-state folding/unfolding transition is visible from the $C_{v}$ plot of $\mathrm{H} \gamma \mathrm{D}$ Crys.

We also estimated the $T_{\mathrm{M}}$ of $\mathrm{Ntd}$ and Ctd domains of both the proteins from the full-length protein simulations by computing the variance in the fraction of native contacts in the $\operatorname{Ntd}\left(\chi_{\mathrm{Ntd}}\right)$ and Ctd domains $\left(\chi_{\mathrm{Ctd}}\right)$ as a function of $T$ (see SI for details). The $T_{M}$ of Ntd and Ctd domains approximately corresponds to the peak position in the plots (Figures S2 and S3). The Ntd and Ctd of $\mathrm{H} \gamma \mathrm{C}$ Crys exhibit differential domain stability with $T_{M}$ of $\mathrm{Ntd}$ and $\mathrm{Ctd} \approx 410 \mathrm{~K}$ and $\approx 420 \mathrm{~K}$, respectively. The $T_{\mathrm{M}}$ of $\mathrm{Ntd}$ and $\mathrm{Ctd}$ domains corresponds to $T_{\mathrm{M}}^{\mathrm{I}}$ and $T_{\mathrm{M}}^{\mathrm{II}}$ of $\mathrm{H} \gamma \mathrm{C}$ Crys in the $C_{v}$ plot (Figure $2 \mathrm{~B}$ ), indicating sequential unfolding of $\mathrm{Ntd}$ followed by $\mathrm{Ctd}$. Thus, the intermediate populated in the $C_{v}$ plot (Figure 2B) of full-length $\mathrm{H} \gamma \mathrm{C}$ Crys consists of a folded Ctd and an unfolded Ntd, evident from the $T_{M}$ values of the individual domains. Unlike $\mathrm{H} \gamma \mathrm{C}$ Crys, truncated $\mathrm{Ntd}$ and $\mathrm{Ctd}$ of $\mathrm{H} \gamma \mathrm{D}$ Crys indicated 
comparable thermal stabilities (with $T_{\mathrm{M}} \approx 426 \mathrm{~K}$ ), in agreement with the $C_{v}$ plot of full-length $\mathrm{H} \gamma \mathrm{D}$ Crys (Figure 1C). However, experiments ${ }^{27}$ report a higher thermodynamic stability of the Ctd $\left(T_{M}=349.2 \mathrm{~K}\right)$ as compared to the $\mathrm{Ntd}$ $\left(T_{\mathrm{M}}=337.5 \mathrm{~K}\right)$ of $\mathrm{H} \gamma \mathrm{D}$ Crys. This anomaly arises due to the reduced-level description of the model systems, as discussed earlier.

FES of $\mathrm{H} \gamma \mathrm{D}$ and $\mathrm{H} \gamma \mathrm{C}$ Crys Folding Show Population of Multiple Intermediate States. Although thermodynamic properties such as $\langle E\rangle$ and $C_{v}$ capture major transitions associated with protein folding/unfolding, they often fail to detect transient intermediates, which occur due to minor structural perturbations that play an important role in protein aggregation. To identify the transient intermediates, we constructed the FES associated with the folding of $\mathrm{H} \gamma \mathrm{D}$ and $\mathrm{H} \gamma \mathrm{C}$ Crys. The FES is projected onto three collective variables (CVs): (1) fraction of native contacts in $\operatorname{Ntd}\left(\chi_{\mathrm{Ntd}}\right),(2)$ fraction of native contacts in Ctd $\left(\chi_{\mathrm{Ctd}}\right)$, and (3) fraction of native contacts at the interface of $\mathrm{Ntd}$ and $\mathrm{Ctd}\left(\chi_{\mathrm{Int}}\right.$; see Methods for details). We employed the $\mathrm{WHAM}^{61}$ to construct the three-dimensional FES, $F\left(\chi_{\text {Ntd }}, \chi_{\text {Ctd }}, \chi_{\text {Int }}\right)$.

The folding FES of $\mathrm{H} \gamma \mathrm{D}$ and $\mathrm{H} \gamma \mathrm{C}$ Crys show that, for both the proteins, at least three distinct minimum energy pathways connect the native $(N)$ and unfolded $(U)$ basins populating different intermediates (Figures 1D,E, 2C,D, S4, and S5). In $\mathrm{H} \gamma \mathrm{D}$ Crys, the most preferred pathway (Path $\mathrm{A}$, shown in black) corresponds to the folding of the Ctd, followed by the folding of Ntd (Figure 1D). In this pathway, four intermediates are populated: (1) a partially folded Ctd with motif $M_{4}$ folded and Ntd unfolded (basin $D_{1}$ ), (2) Ctd completely folded and $\mathrm{Ntd}$ unfolded (basin $D_{2}$ ), (3) Ctd completely folded and motif $M_{1}$ of Ntd folded (basin $D_{3}$ ), and (4) both Ctd and Ntd folded with disrupted interdomain contacts (basin $I^{*}$ ) (Figures $\mathrm{S} 4 \mathrm{~A}, \mathrm{~B}, \mathrm{C}, \mathrm{H}$ and $\mathrm{S} 6 \mathrm{~A}$ ). Intermediate $\mathrm{D}_{2}$ with a folded Ctd and unfolded $\mathrm{Ntd}$ was inferred from experiments ${ }^{9,22}$ and was also observed in the $\mathrm{H} \gamma \mathrm{D}$ unfolding simulations. ${ }^{13,15}$

In the second preferred pathway (Path B, shown in red), $\mathrm{Ntd}$ folds prior to the folding of Ctd (Figure 1D). In this pathway, four intermediates are populated: (1) a partially folded Ntd with motif $M_{1}$ folded and Ctd unfolded (basin $D_{4}$ ), (2) completely folded Ntd and unfolded Ctd (basin $D_{5}$ ), (3) completely folded $\mathrm{Ntd}$ and partially folded Ctd with motif $M_{4}$ folded (basin $D_{6}$ ), and (4) intermediate $I^{*}$, which is also common to Path A (Figure S4D-F). AFM experiments on $\mathrm{H} \gamma \mathrm{D}$ Crys provide evidence for the population of an intermediate with a folded $\mathrm{Ntd}$ and unfolded $\mathrm{Ctd}$, which is similar to the intermediate, $D_{5}$. Interestingly, kinetic unfolding experiments on $\mathrm{H} \gamma \mathrm{D}$ Crys in the absence of denaturants indicated higher kinetic stability of $\mathrm{Ntd}$ compared to $\mathrm{Ctd} .{ }^{11}$

In Paths $\mathrm{A}$ and $\mathrm{B}$, sequential folding of the domains is observed. Whereas in the third folding pathway (Path $\mathrm{C}$, shown in green), which is the least favored, an intermediate with both the domains partially folded is observed. An intermediate with partially folded Ntd with motif $M_{1}$ folded and partially folded Ctd with motif $M_{4}$ folded (basin $D_{7}$ ) is populated in addition to $D_{4}, D_{3}$, and $I^{*}$ (Figures $S 4 G$ and S6A).

Similar to $\mathrm{H} \gamma \mathrm{D}$ Crys, the preferred path connecting the $N$ and $U$ basins in $\mathrm{H} \gamma \mathrm{C}$ Crys (Path A shown in black) involves stepwise folding of the Ctd prior to the folding of Ntd. The four intermediates populated in this path are (1) partially folded Ctd with motif $M_{4}$ folded and unfolded Ntd (basin $C_{1}$ ), (2) completely folded Ctd and unfolded Ntd (basin $C_{2}$ ), (3) completely folded Ctd and partially folded Ntd with $M_{1}$ folded (basin $C_{3}$ ), and (4) both Ctd and $\mathrm{Ntd}$ folded with disrupted interdomain contacts $\left(I^{*}\right.$; Figures $2 \mathrm{C}, \mathrm{S} 5 \mathrm{~A}, \mathrm{~B}, \mathrm{C}, \mathrm{H}$, and $\left.\mathrm{S} 6 \mathrm{~B}\right)$. In the second preferred path (path B shown in red), Ntd folds prior to Ctd. The four intermediates populated in this path are (1) partially folded Ntd with folded motif $M_{1}$ and unfolded Ctd (basin $C_{4}$ ), (2) completely folded Ntd and unfolded Ctd (basin $C_{5}$ ), (3) completely folded Ntd and partially folded Ctd with motif $M_{4}$ folded (basin $C_{6}$ ), and (4) intermediate $I^{*}$ (Figure S5D-F and S6B). The least-favored path (path C shown in green) involves an intermediate with a partially structured Ntd with motif $M_{1}$ folded and partially structured Ctd with motif $M_{4}$ folded (basin $C_{7}$ ) in addition to $C_{3}, C_{4}$, and $I^{*}$ (Figures S5G and S6B).

The intermediate, $I^{*}$, which is common to all the folding pathways of $\mathrm{H} \gamma \mathrm{D}$ and $\mathrm{H} \gamma \mathrm{C}$ Crys is populated due to structural fluctuations in the native state ensemble. A hydrophobic patch at the domain interface of $\mathrm{Ntd}$ and $\mathrm{Ctd}$ gets exposed when $I^{*}$ is populated, and this makes it an ideal candidate for domain swapping. Human $\beta \mathrm{B}_{2}$ Crys, which belongs to the $\beta \gamma$ Crys superfamily, forms such domain-swapped dimers by swapping the $\mathrm{Ntd}$ and $\mathrm{Ctd}$ while the linker region acts as the hinge loop. ${ }^{51}$ Similar domain-swapped oligomers are also found in mouse $\gamma S$ Crys, which is a member of the $\gamma$ Crys family. Although no such domain-swapped structures are reported for $\mathrm{H} \gamma \mathrm{D}$ and $\mathrm{H} \gamma \mathrm{C}$ Crys, intermediate $I^{*}$ can potentially lead to such domain-swapped oligomers. A recent computational study on the W42R mutant of $\mathrm{H} \gamma \mathrm{D}$ Crys showed the presence of $I^{*}$ intermediates with exposed interdomain hydrophobic interface, which led to large scale aggregation involving NtdNtd interactions. ${ }^{62}$

The rest of the partially structured intermediate states populated in the folding pathways of $\mathrm{H} \gamma \mathrm{D}$ and $\mathrm{H} \gamma \mathrm{C}$ Crys can act as potential precursors to aggregation as well. The formation of sticky hydrophobic patches on the protein surface has been related to aggregation in cataract associated mutants of $\mathrm{H} \gamma \mathrm{D}$ Crys. ${ }^{53,63,64}$ Experimental studies on $\mathrm{H} \gamma \mathrm{D}$ Crys indicated the presence of intermediates with either Ctdfolded (basin $\left.D_{2}\right)^{9,22}$ or Ntd-folded (basin $\left.D_{5}\right) \cdot{ }^{11,12}$ However, the role of these intermediates in aggregation remains unclear. The domain-swapped dimer structure of $\mathrm{H} \gamma \mathrm{D}$ Crys was predicted in AFM experiments, where $\beta_{1} \beta_{2}$ strands from $\mathrm{Ntd}$ get swapped between neighboring monomers. ${ }^{12}$ Further, fluorescence spectra of the chaperone ( $\alpha \mathrm{B}$ Crys) bound complex of $\mathrm{H} \gamma \mathrm{D}$ Crys showed that both of the domains were partially unstructured in the substrate, ${ }^{44}$ unlike the experimentally observed folding intermediates, which consist of a folded domain. Intermediates, such as $D_{7}$ and $C_{7}$ populated in the FES of $\mathrm{H} \gamma \mathrm{D}$ and $\mathrm{H} \gamma \mathrm{C}$ Crys, consist of partially unfolded $\mathrm{Ntd}$ and $\mathrm{Ctd}$ and meet the requirements for possible aggregation prone precursors. These intermediates or structurally related intermediates with an exposed hydrophobic interface might play an important role in the aggregation of $\gamma$ Crys proteins.

Dimerization in $\mathrm{H} \gamma \mathrm{D}$ and $\mathrm{H} \gamma \mathrm{C}$ Crys. Surgically removed cataractous lenses contain high molecular weight aggregates of partially folded $\gamma$ Crys. $^{26,44,65}$ However, the specific conformations of the proteins in the aggregates remain elusive due to the insoluble nature of the aggregates. A detailed understanding of the misfolded conformations is crucial to elucidate the underlying molecular mechanism of cataract formation and design essential therapeutics. To understand the initial molecular interactions that advance to cataract 

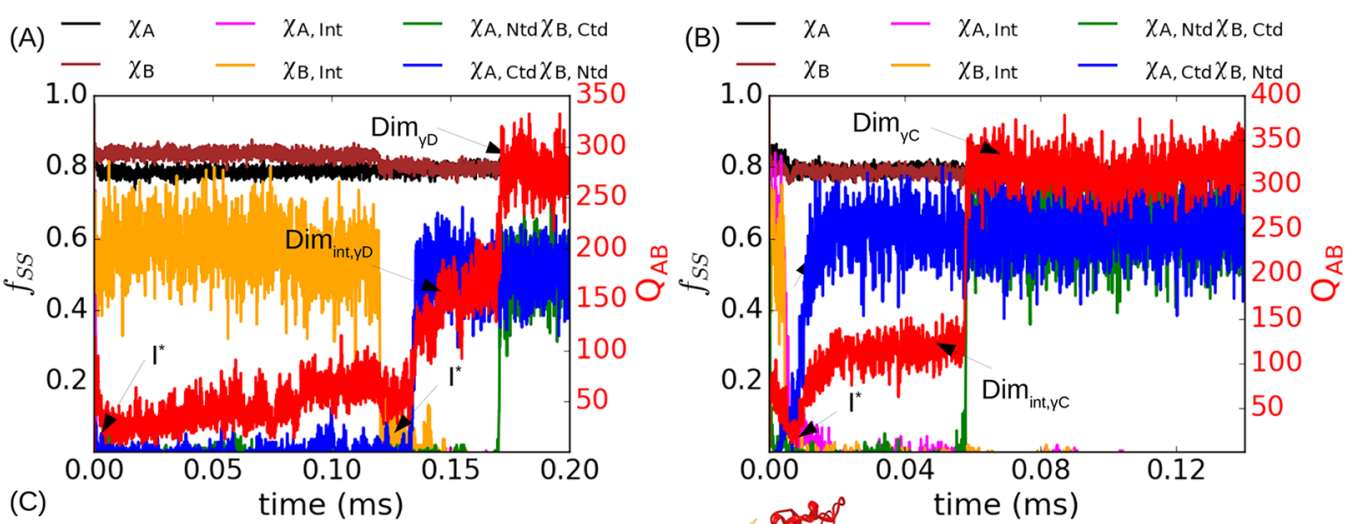

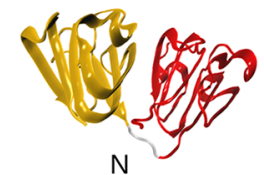

time $=0 \mathrm{~ms}$

(D)

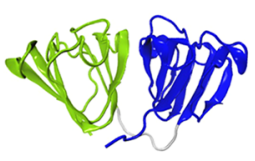

N

time $=0 \mathrm{~ms}$

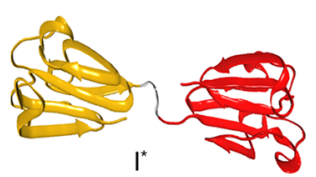

time $\approx 0.00 \mathrm{~ms}, 0.13 \mathrm{~ms}$

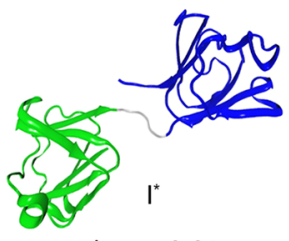

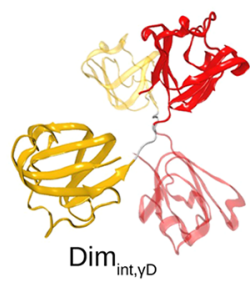

time $\approx 0.15 \mathrm{~ms}$

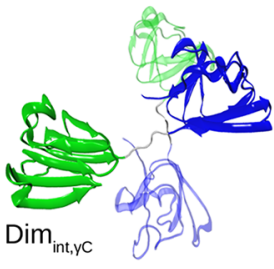

time $\approx 0.02 \mathrm{~ms}$

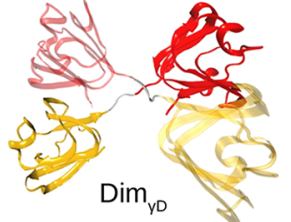

time $\geq 0.17 \mathrm{~ms}$

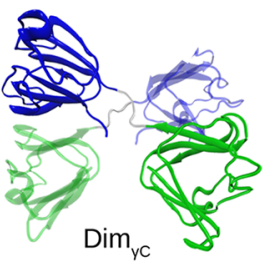

time $\geq 0.05 \mathrm{~ms}$

Figure 3. Dimerization from the native states of $\mathrm{H} \gamma \mathrm{D}$ and $\mathrm{H} \gamma \mathrm{C}$ Crys at $T=300 \mathrm{~K}$. (A, B) Time evolution of the fraction of secondary structural contacts $\left(f_{\mathrm{ss}}\right)$ present in the two monomers $\mathrm{A}$ and $\mathrm{B}$, and the total number of native contacts $Q_{\mathrm{AB}}$ formed between $\mathrm{A}$ and $\mathrm{B}$. Dimerization takes place in two steps. In the first step, only one domain gets swapped $\left(\operatorname{Dim}_{\text {int, } \gamma \mathrm{D}}, \mathrm{Dim}_{\mathrm{int}, \gamma \mathrm{C}}\right)$, while in the second step, both the domains are swapped $\left(\operatorname{Dim}_{\gamma \mathrm{D}}, \operatorname{Dim}_{\gamma \mathrm{C}}\right)$. Mechanism of dimerization in $\mathrm{H} \gamma \mathrm{D}(\mathrm{C})$ and $\mathrm{H} \gamma \mathrm{C}$ Crys (D). An intermediate state $I^{*}$ with disrupted interdomain contacts get populated in the initial stages of dimerization, and its formation is stochastic. $I^{*}$ further swaps the Ntd and Ctd with neighboring monomers in two stages leading to domain-swapped dimers in $26 \%$ of $\mathrm{H} \gamma \mathrm{D}$ and $4 \%$ of $\mathrm{H} \gamma \mathrm{C}$ Crys dimerization trajectories.

formation in later stages, we studied the formation of dimers, which are the simplest possible oligomers, using symmetric Go potential to model intermonomer interactions ${ }^{32,58}$ (Figure S1).

Dimerization from the Native State. $\gamma$ Crys are generally monomeric in solution. However, octameric structures of mouse $\gamma S$ Crys are published in the PDB, where Ntds and Ctds are swapped between the neighboring monomers with linker region acting as hinge loop (PDB ID: $6 \mathrm{MYG}$ and $6 \mathrm{MYH}$ ), indicating oligomerization in the native state of the protein. Similar domain swapping is also observed in human $\beta \mathrm{B}_{2}$ Crys, ${ }^{51}$ which belongs to the $\beta \gamma$ Crys superfamily. To elucidate whether such oligomerization is also feasible in the native states of $\mathrm{H} \gamma \mathrm{D}$ and $\mathrm{H} \gamma \mathrm{C}$ Crys, we performed 50 independent Brownian dynamics simulations starting from two-folded monomers (say monomers A and B) separated by $20 \AA$ ( $\approx R_{\mathrm{g}}$ of a monomer) using a harmonic restraint with a spring constant, $k=2.0 \mathrm{kcal} / \mathrm{mol} / \AA^{2}$ at $T=$ $300 \mathrm{~K}$. To track the progress of dimerization, we computed the number of contacts $Q_{A B}$ formed between the monomers $A$ and $\mathrm{B}$ as a function of simulation time (Figure 3A,B). A two-step dimerization process was observed only in a fraction of independent Brownian dynamics trajectories (26\% in $\mathrm{H} \gamma \mathrm{D}$ and $4 \%$ in $\mathrm{H} \gamma \mathrm{C}$ Crys $)$ yielding unique dimer structures $\left(\operatorname{Dim}_{\gamma \mathrm{D}}\right.$ and $\left.\operatorname{Dim}_{\gamma \mathrm{C}}\right)$, while in the rest of the trajectories, proteins folded back to their native states. A two-step dimerization process was observed in both the proteins yielding unique dimer structures $\left(\operatorname{Dim}_{\gamma \mathrm{D}}\right.$ and $\left.\operatorname{Dim}_{\gamma \mathrm{C}}\right)$.
In the final dimer structure, both Ntd and Ctd of the two monomers remain folded (fraction of native contacts in monomer $\mathrm{A}, \chi_{\mathrm{A}} \approx 1$ and in monomer $\mathrm{B}, \chi_{\mathrm{B}} \approx 1$ ); however, the interdomain contacts present between $\mathrm{Ntd}$ and Ctd of each monomer get disrupted $\left(\chi_{\mathrm{A}, \text { Int }} \approx 0, \chi_{\mathrm{B}, \text { Int }} \approx 0\right.$; Figure 3A,B). Instead, two new interfaces form between $\mathrm{Ntd}$ of monomer $\mathrm{A}$ and $\mathrm{Ctd}$ of monomer $\mathrm{B}\left(\chi_{\mathrm{A}, \mathrm{Ntd} ; \mathrm{B}, \mathrm{Ctd}} \approx 1\right)$, and $\mathrm{Ctd}$ of monomer $\mathrm{A}$ and $\mathrm{Ntd}$ of monomer $\mathrm{B}\left(\chi_{\mathrm{A}, \mathrm{Ctd} ; \mathrm{B}, \mathrm{Ntd}} \approx 1\right)$. This reveals a domain-swapping mechanism, where neighboring monomers exchange Ntd and Ctd with each other forming two new interfaces, while the linker region acts as hinge loop. Dimers $\operatorname{Dim}_{\gamma \mathrm{D}}$ and $\operatorname{Dim}_{\gamma \mathrm{C}}$ obtained for $\mathrm{H} \gamma \mathrm{D}$ and $\mathrm{H} \gamma \mathrm{C}$ Crys, respectively, are very similar to the domain-swapped structure obtained for mouse $\gamma$ S Crys (PDB ID: 6MYG, 6MYH). NMR studies $^{66}$ further show that amorphous aggregates of cataractassociated P23T mutant of $\mathrm{H} \gamma \mathrm{D}$ Crys retains the native fold, indicating native-like conformations taking part in aggregation.

In the first step of dimerization, proteins break their interdomain contacts exposing the hydrophobic pocket present at the interface, populating the intermediate $I^{*}$. In $I^{*}$, the linker region adopts an extended conformation, which facilitates domain swapping (Figure 3C,D). In the next step, monomers swap either $\mathrm{Ntd}$ or $\mathrm{Ctd}$ with each other leading to half-swapped dimers $\operatorname{Dim}_{\text {int }, \gamma \mathrm{D}}$ and $\operatorname{Dim}_{\mathrm{int}, \gamma \mathrm{C}}$. Finally the remaining domains are swapped between the monomers resulting in fully swapped dimers $\operatorname{Dim}_{\gamma \mathrm{D}}$ and $\operatorname{Dim}_{\gamma \mathrm{C}}$. Intermediate $I^{*}$, which is key to dimerization in the native 
state gets populated during the initial stages of unfolding of both the proteins (Figures 1D and 2C). External factors such as low denaturant concentrations or mildly elevated temperatures should favor the formation of $I^{*}$, which can take part in domain swapping with neighboring monomers leading to the formation of oligomeric structures. We speculate that mild protein denaturing conditions can increase the propensity for domain swapping in Crys proteins. The resulting dimeric and oligomeric moieties with multiple interprotein interfaces can resist unfolding to relatively higher denaturant concentrations and lead to hysteresis, as observed in experiments. ${ }^{10,23,24}$

Dimerization During Refolding. Aggregation is also observed experimentally for $\mathrm{H} \gamma \mathrm{D}$ and $\mathrm{H} \gamma \mathrm{C}$ Crys when $[\mathrm{GdHCl}]$ is lowered from high values to less than $1 \mathrm{M}^{10}$ Aggregation suppression experiments on $\gamma$ Crys using chaperone $\alpha \mathrm{B}$ Crys further identified partially unfolded substrates of $\mathrm{H} \gamma \mathrm{C}, \mathrm{H} \gamma \mathrm{D}$, and $\mathrm{H} \gamma \mathrm{S}$ Crys in the aggregates. ${ }^{44}$ It was further concluded that both $\mathrm{Ntd}$ and $\mathrm{Ctd}$ of $\mathrm{H} \gamma \mathrm{D}$ Crys must be partially unfolded for in vitro aggregation. ${ }^{44}$ To identify conformations of $\mathrm{H} \gamma \mathrm{D}$ and $\mathrm{H} \gamma \mathrm{C}$ Crys populated during refolding that result in aggregation, we studied dimerization of the proteins during refolding from unfolded conformations at $T=300 \mathrm{~K}$. We performed 100 independent Brownian dynamics simulations starting from different unfolded conformations of the monomers separated by $\approx 20$ $\AA$. Dimerization was observed in $\approx 60 \%$ of the trajectories in $\mathrm{H} \gamma \mathrm{D}$ and $\mathrm{H} \gamma \mathrm{C}$ Crys.

An ensemble of domain-swapped dimer structures were identified, where at least in $45 \%$ of the structures of $\mathrm{H} \gamma \mathrm{D}$ Crys $(\approx 40 \%$ of $\mathrm{H} \gamma \mathrm{C}$ Crys $)$, secondary structural units were swapped from both $\mathrm{Ntd}$ and $\mathrm{Ctd}$, resulting in double domain-swapped dimers. In the rest of the structures, either $\mathrm{Ntd}(\approx 40 \%$ in $\mathrm{H} \gamma \mathrm{D}$ Crys, $\approx 37 \%$ in $\mathrm{H} \gamma \mathrm{C}$ Crys) or Ctd $(\approx 15 \%$ in $\mathrm{H} \gamma \mathrm{D}$ Crys, $\approx 23 \%$ in $\mathrm{H} \gamma \mathrm{C}$ Crys) took part in swapping, while the other domain folded to its native structure. In both the proteins, most populated double domain-swapped dimers are formed by swapping the secondary structural units from both Ntd and Ctd. The primary swapping element in the $\mathrm{Ntds}$ is the first hairpin loop $\beta_{1} \beta_{2}$ from motif $M_{1}$ (Figures 4A,C and S7A,C). Swapping of $\beta_{1} \beta_{2}$ strands was predicted in an AFM study of $\mathrm{H} \gamma \mathrm{D}$ Crys. ${ }^{12}$ In Ctds (Figures $4 \mathrm{~B}, \mathrm{C}$ and S7B,C), the primary swapping units are the first hairpin loop of motif $M_{3}\left(\beta_{6} \beta_{7}\right.$ in $\mathrm{H} \gamma \mathrm{D}$ Crys and $\beta_{8} \beta_{9}$ in $\mathrm{H} \gamma \mathrm{C}$ Crys).

In $\approx 10 \%$ of the cases, we observed domain-swapped structures similar to $\operatorname{Dim}_{\gamma \mathrm{D}}$ and $\operatorname{Dim}_{\gamma \mathrm{C}}$, where both the domains are completely swapped between the monomers. Other less common swapping units (with a population $<5 \%$ ) were identified in $\mathrm{H} \gamma \mathrm{D}$ and $\mathrm{H} \gamma \mathrm{C}$ Crys, which led to both single and double domain-swapped dimer structures (Figures S8 and S9). Ntd swapping units include $\beta_{1}, \beta_{3} \beta_{4}$, and motif $M_{1}$ in $\mathrm{H} \gamma \mathrm{D}$ Crys, and $\beta_{1}, \beta_{7}$, and motif $M_{1}$ in $\mathrm{H} \gamma \mathrm{C}$ Crys. Ctd swapping units include $\beta_{6}$ and $\beta_{12}$ in $\mathrm{H} \gamma \mathrm{D}$ Crys, and $\beta_{8}$ and $\beta_{14}$ in $\mathrm{H} \gamma \mathrm{C}$ Crys. Some of the intermediates populated in the folding FES of $\mathrm{H} \gamma \mathrm{D}\left(D_{3}, D_{4}, D_{7}\right)$ and $\mathrm{H} \gamma \mathrm{C}$ Crys $\left(C_{3}, C_{4}, C_{7}\right)$ with a folded motif $M_{1}$ should facilitate swapping of $M_{1}$ between the Ntds as observed in the ensemble of swapped dimer structures.

In the most populated dimers, domain swapping took place after formation of the $\beta$ hairpin loops $\beta_{1} \beta_{2}, \beta_{6} \beta_{7}$ (in $\mathrm{H} \gamma \mathrm{D}$ Crys), and $\beta_{8} \beta_{9}$ (in $\mathrm{H} \gamma \mathrm{C}$ Crys) (Figures 4 and S7). In the final domain-swapped structures, the $\beta_{1}$ strand makes contact with the $\beta_{3}^{\prime}$ strands from the other monomer and vice versa. On the other hand, in Ctds, the $\beta_{6}$ strand in the $\mathrm{H} \gamma \mathrm{D}$ Crys $\left(\beta_{8}\right.$ strand in $\mathrm{H} \gamma \mathrm{C}$ Crys) makes contact with the $\beta_{8}^{\prime}$ strand ( $\beta_{10}^{\prime}$ strand in
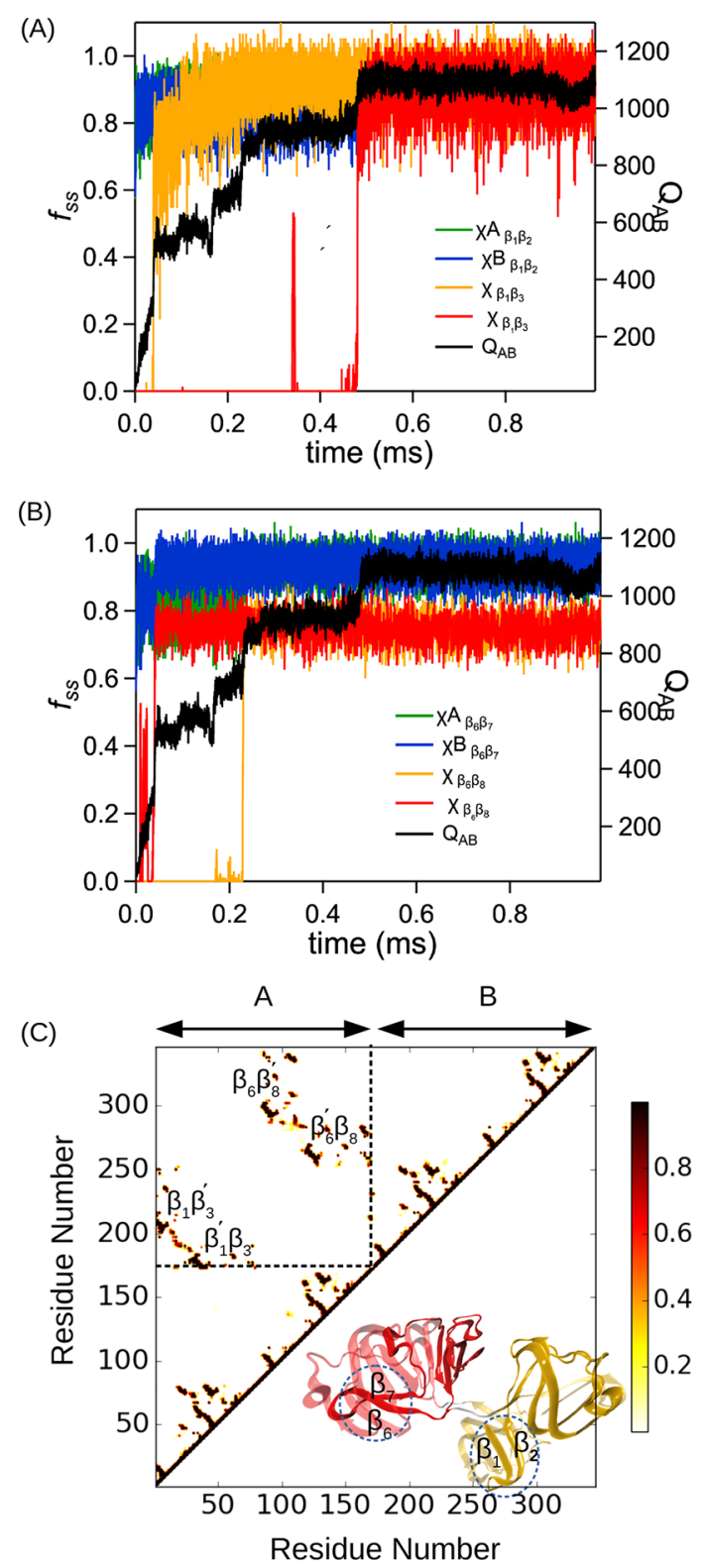

Figure 4. Multistep dimerization in $\mathrm{H} \gamma \mathrm{D}$ Crys while refolding at $T=$ $300 \mathrm{~K}$. Secondary structural elements from $\mathrm{Ntd}\left(\beta_{1} \beta_{2}\right)$ and $\mathrm{Ctd}$ $\left(\beta_{6} \beta_{7}\right)$ get swapped leading to a double domain-swapped dimer. (A) Time evolution of a fraction of secondary structural contacts between $\beta_{1} \beta_{2}$ strands in monomers $\mathrm{A}$ and $\mathrm{B}\left(\chi_{\beta_{1} \beta_{2}}^{\mathrm{A}}, \chi_{\beta_{1} \beta_{2}}^{\mathrm{B}}\right)$ and between $\beta_{1}$ strand of monomer $\mathrm{A}$ and $\beta_{3}^{\prime}$ strand of monomer $\mathrm{B}\left(\chi_{\beta_{1} \beta_{3}^{\prime}}\right)$ and vice versa $\left(\chi_{\beta_{1} \beta_{3}}\right)$, along with the number of contacts present between monomers $\mathrm{A}$ and $\mathrm{B}\left(Q_{\mathrm{AB}}\right)$. (B) Time evolution of a fraction of secondary structural contacts between $\beta_{6} \beta_{7}$ strands in monomers $\mathrm{A}$ and $\mathrm{B}\left(\chi_{\beta_{6} \beta_{7}}^{\mathrm{A}}\right.$, $\left.\chi_{\beta_{6} \beta_{7}}^{\mathrm{B}}\right)$ and between the $\beta_{6}$ strand of monomer $\mathrm{A}$ and the $\beta_{8}^{\prime}$ strand of monomer B $\left(\chi_{\beta_{6} \beta_{8}^{\prime}}\right)$ and vice versa $\left(\chi_{\beta_{6} \beta_{8}^{\prime}}\right)$. (C) Contact map of the final domain-swapped dimer structure. $\beta_{1}$ strand makes contacts with the $\beta_{3}^{\prime}$ strand of the other monomer and vice versa after swapping of $\beta_{1} \beta_{2}$ strands. The first hairpin loop of $M_{3}\left(\beta_{6} \beta_{7}\right)$ gets swapped between the Ctds where the $\beta_{6}$ strand forms a contact with the $\beta_{8}^{\prime}$ strand from the other monomer and vice versa.

$\mathrm{H} \gamma \mathrm{C}$ Crys) from the other monomer and vice versa. Interestingly, in the $\mathrm{H} \gamma \mathrm{D}$ Crys, the highest number of pathogenic mutations are located in the $\beta_{1} \beta_{2}$ hairpin loop. . $^{4,52-55}$ The double domain-swapping mechanism re- 
ported in this study can be used to explain higher order oligomer formation in $\gamma$ Crys where instead of reciprocal domain swapping, secondary structures can be exchanged with neighboring monomers in a chain-like manner.

\section{CONCLUSIONS}

Cataract-associated misfolding and aggregation mechanism of eye lens Crys proteins remains largely unknown. Using coarsegrained models and molecular dynamics simulations, we explored the folding pathway and dimerization of $\mathrm{H} \gamma \mathrm{D}$ and $\mathrm{H} \gamma \mathrm{C}$ Crys. The multidimensional FES shows three competing folding pathways with distinct intermediates. In the two dominant pathways, the domains sequentially fold, populating intermediates with either Ntd or Ctd folded. The third, leastfavored pathway populates intermediates with partially structured Ntd and Ctd, which can facilitate aggregation from both the domains. Interestingly, fluorescence spectra of chaperone ( $\alpha \mathrm{B}$ Crys) bound complex of $\mathrm{H} \gamma \mathrm{D}$ Crys indicated that both the domains are partially structured in the aggregation prone conformations. ${ }^{44}$ All three pathways populate a native-like intermediate state $\left(I^{*}\right)$, with two folded domains and disrupted interdomain contacts. Under native-like conditions, $I^{*}$ forms domain-swapped dimers by exchanging folded domains with other monomers. Such oligomerization, if takes place during early stages of unfolding, may lead to hysteresis in the folding experiments. Similar domain-swapped structures are reported for human $\beta \mathrm{B} 2 \mathrm{Crys}^{49-51}$ and mouse $\gamma \mathrm{S}$ Crys.

We have also identified an ensemble of domain-swapped dimers from the dimerization simulations performed using unfolded conformations of $\mathrm{H} \gamma \mathrm{D}$ and $\mathrm{H} \gamma \mathrm{C}$ Crys, which mimic aggregation during refolding. The $\mathrm{Ntd}$ swapping units $\left(\beta_{1} \beta_{2}\right)$ for $\mathrm{H} \gamma \mathrm{D}$ Crys were already hinted in previous studies ${ }^{12,43,67}$ and contain the highest number of pathogenic mutations. We also identified swapping units in the $\mathrm{Ctds}\left(\beta_{6} \beta_{7}\right.$ in $\mathrm{H} \gamma \mathrm{D}$ and $\beta_{8} \beta_{9}$ in $\mathrm{H} \gamma \mathrm{C}$ Crys), which in conjunction with Ntd swapping units led to double domain-swapped dimers. Other less populated dimers involved the swapping of different secondary structures from the $\mathrm{Ntds}$ and the Ctds, leading to heterogeneity in the dimer structures. The double domainswapping mechanism presented in this study provides structural insights to the early oligomeric assembies in $\mathrm{H} \gamma \mathrm{D}$ and $\mathrm{H} \gamma \mathrm{C}$ Crys. Knowledge of the swapping units present in the two domains can be exploited to design preventive measures and essential therapeutics for cataracts.

The coarse-grained model used in this study has been successfully applied to elucidate folding ${ }^{48,68,69}$ and aggregation of other proteins. ${ }^{70-72}$ However, the native-centric nature of this model limits the incorporation of non-native interactions that might play a crucial role in protein aggregation. Due to the low resolution of a coarse-grained model, the atom level description of protein-protein and solvent-protein interactions are also beyond the scope of this model. In the SOP-SC model used in this work, we have not incorporated features that allow the formation of non-native disulfide bonds. However, such disulfide bonds have been proven to be very important in the formation of Crystallin aggregates.

Age-related nuclear cataracts often result from the oxidative stress built up in an aged lens due to gradual depletion in the glutathione (GSH) levels. ${ }^{73,74}$ Due to oxidative stress, Cys residues form disulfide links, which are found commonly in mature cataractous lenses. ${ }^{75-78}$ Consequently, the impact of disulfide bonds in the aggregation of Crys proteins, especially in Cys-rich $\gamma$ Crys is the subject of intense investigation. ${ }^{43,67,79-81}$ A recent experiment ${ }^{67}$ provided evidence for the dynamic transfer of disulfide bonds from oxidized WT $\mathrm{H} \gamma \mathrm{D}$ Crys to a mutant W42Q promoting aggregation in the latter. Another experimental study ${ }^{43}$ in conjunction with simulations identified an internal non-native disulfide bond (Cys32-Cys41), which traps an aggregation prone conformation, essential for oxidative aggregation of mutant variants (W42R, W42Q) of $\mathrm{H} \gamma \mathrm{D}$ Crys. The trapped intermediate obtained from the mutant simulations, features a detached $\mathrm{N}$ terminal $\beta$-hairpin. This intermediate is structurally similar to the intermediate predicted in AFM experiments ${ }^{12}$ of WT H $\gamma \mathrm{D}$ Crys and also consistent with the domain-swapped dimer structures predicted in this work for the WT protein. This shows that aggregation prone intermediates, which are predominantly populated by mutants, are transiently populated in WT proteins as well. In our future computational studies, we will probe the role of non-native disulfide bonds in the population of aggregation-prone structures by $\gamma$ Crys proteins using coarse-grained protein models, ${ }^{82,83}$ which allow the formation of such bonds.

\section{ASSOCIATED CONTENT}

\section{Supporting Information}

The Supporting Information is available free of charge at https://pubs.acs.org/doi/10.1021/acs.jpcb.0c07833.

Detailed description of the coarse-grained SOP-SC model, symmetric GO potential, Langevin and Brownian dynamics simulations; illustration of the GO potential in a dimeric system (Figure S1); illustration of a two state folding of the truncated domains of $\mathrm{H} \gamma \mathrm{C}$ (Figure S2) and $\mathrm{H} \gamma \mathrm{D}$ (Figure S3) Crys; contact maps and representative structures of the intermediates populated in equilibrium simulations of $\mathrm{H} \gamma \mathrm{D}$ (Figure S4) and $\mathrm{H} \gamma \mathrm{C}$ (Figure S5) Crys; minimum energy pathways connecting the folded and unfolded states in the twodimensional FES of $\mathrm{H} \gamma \mathrm{D}$ and $\mathrm{H} \gamma \mathrm{C}$ Crys (Figure S6); illustration of the multistep dimerization in $\mathrm{H} \gamma \mathrm{C}$ Crys while refolding (Figure S7); contact maps and representative structures of the less-populated dimers of $\mathrm{H} \gamma \mathrm{D}$ (Figure S8) and $\mathrm{H} \gamma \mathrm{C}$ (Figure S9) Crys during refolding; energy function parameters for the SOP-SC model (Table S1); SOP-SC model parameters for monomer and dimer simulations (Table S2); sidechain radii of amino acids (Table S3) (PDF)

\section{AUTHOR INFORMATION}

\section{Corresponding Author}

Govardhan Reddy - Solid State and Structural Chemistry Unit, Indian Institute of Science, Bengaluru, Karnataka, India 560012; 이이.org/0000-0002-9013-8040; Phone: +9180-22933533; Email: greddy@iisc.ac.in; Fax: +91-8023601310

\section{Authors}

Balaka Mondal - Solid State and Structural Chemistry Unit, Indian Institute of Science, Bengaluru, Karnataka, India 560012

Jayashree Nagesh - Solid State and Structural Chemistry Unit, Indian Institute of Science, Bengaluru, Karnataka, India 560012; ○ orcid.org/0000-0002-0415-8329

Complete contact information is available at: 
https://pubs.acs.org/10.1021/acs.jpcb.0c07833

\section{Notes}

The authors declare no competing financial interest.

\section{ACKNOWLEDGMENTS}

G.R. is grateful to Prof. D. Thirumalai for his mentoring and support. G.R. acknowledges funding from the National Supercomputing Mission (NSM) through the Grant MeitY/ R\&D/HPC/2(1)/2014. J.N. thanks DST-SERB for funding under the Ramanujan Faculty Fellowship. B.M. acknowledges the research fellowship from Indian Institute of ScienceBangalore. The computations are performed using the TUE and Cray XC40 cluster at IISc.

\section{REFERENCES}

(1) Moreau, K. L.; King, J. A. Protein misfolding and aggregation in cataract disease and prospects for prevention. Trends Mol. Med. 2012, $18,273-282$.

(2) Lampi, K.; Ma, Z.; Shih, M.; Shearer, T.; Smith, J.; Smith, D.; David, L. Sequence analysis of $\beta \mathrm{A} 3, \beta \mathrm{B} 3$, and $\beta \mathrm{A} 4$ crystallins completes the identification of the major proteins in young human lens. J. Biol. Chem. 1997, 272, 2268-2275.

(3) Robinson, N. E.; Lampi, K. J.; Speir, J. P.; Kruppa, G.; Easterling, M.; Robinson, A. B. Quantitative measurement of young human eye lens crystallins by direct injection Fourier transform ion cyclotron resonance mass spectrometry. Mol. Vis. 2006, 12, 704-711.

(4) Horwitz, J. $\alpha$-Crystallin can function as a molecular chaperone. Proc. Natl. Acad. Sci. U. S. A. 1992, 89, 10449-10453.

(5) Delaye, M.; Tardieu, A. Short-range order of Crystallin proteins accounts for eye lens transparency. Nature 1983, 302, 415-417.

(6) Stradner, A.; Foffi, G.; Dorsaz, N.; Thurston, G.; Schurtenberger, P. New insight into cataract formation: enhanced stability through mutual attraction. Phys. Rev. Lett. 2007, 99, 198103.

(7) Siezen, R.; Thomson, J.; Kaplan, E.; Benedek, G. Human lens $\gamma$ crystallins - isolation, identification, and characterization of the expressed gene-products. Proc. Natl. Acad. Sci. U. S. A. 1987, 84, 6088-6092.

(8) Slingsby, C.; Clout, N. Structure of the crystallins. Eye 1999, 13, 395-402.

(9) Flaugh, S.; Kosinski-Collins, M.; King, J. Interdomain side-chain interactions in human $\gamma \mathrm{D}$ Crystallin influencing folding and stability. Protein Sci. 2005, 14, 2030-2043.

(10) Kosinski-Collins, M.; King, J. In vitro unfolding, refolding, and polymerization of human $\gamma \mathrm{D}$ Crystallin, a protein involved in cataract formation. Protein Sci. 2003, 12, 480-490.

(11) Henry, I. M.; Kosinski-Collins, M.; Thol, S.; Serebryany, E.; King, J. A. Kinetic stability of long-lived human $\gamma \mathrm{D}$ and $\gamma \mathrm{S}$ lens crystallins, derived in part from their domain interfaces, may protect against cataract. Biophys. J. 2019, 116, 321A-322A.

(12) Garcia-Manyes, S.; Giganti, D.; Badilla, C. L.; Lezamiz, A.; Perales-Calvo, J.; Beedle, A. E. M.; Fernandez, J. M. Single-molecule force spectroscopy predicts a misfolded, domain-swapped conformation in human $\gamma$ D-Crystallin protein. J. Biol. Chem. 2016, 291, 42264235.

(13) Das, P.; King, J. A.; Zhou, R. $\beta$-Strand interactions at the domain interface critical for the stability of human lens $\gamma \mathrm{D}$-Crystallin. Protein Sci. 2009, 19, 131-140.

(14) Das, P.; King, J. A.; Zhou, R. Aggregation of $\gamma$-crystallins associated with human cataracts via domain swapping at the Cterminal $\beta$-strands. Proc. Natl. Acad. Sci. U. S. A. 2011, 108, 1051410519.

(15) Sikora, M.; Cieplak, M. Mechanical stability of multidomain proteins and novel mechanical clamps. Proteins: Struct., Funct., Genet. 2011, 79, 1786-1799.
(16) Itoh, K.; Sasai, M. Cooperativity, connectivity, and folding pathways of multidomain proteins. Proc. Natl. Acad. Sci. U. S. A. 2008, 105, 13865-13870.

(17) Batey, S.; Nickson, A. A.; Clarke, J. Studying the folding of multidomain proteins. HFSP J. 2008, 2, 365-377.

(18) Itoh, K.; Sasai, M. Multidimensional theory of protein folding. J. Chem. Phys. 2009, 130, 145104.

(19) Naveenkumar, N.; Kumar, G.; Srinivasan, N.; Sowdhamini, R.; Vishwanath, S. Fold combinations in multi-domain proteins. Bioinformation 2019, 15, 342-350.

(20) Fu, L.; Liang, J. Unfolding of human lens recombinant $\beta$ B2and $\gamma$ C-crystallins. J. Struct. Biol. 2002, 139, 191-198.

(21) Fu, L.; Liang, J. Spectroscopic analysis of lens recombinant $\beta \mathrm{B} 2-$ and $\gamma \mathrm{C}$-Crystallin. Mol. Vis. 2001, 7, 178-183.

(22) Kosinski-Collins, M.; Flaugh, S.; King, J. Probing folding and fluorescence quenching in human $\gamma \mathrm{D}$ Crystallin greek key domains using triple tryptophan mutant proteins. Protein Sci. 2004, 13, 22232235.

(23) Flaugh, S. L. Contributions of hydrophobic domain interface interactions to the folding and stability of human $\gamma \mathrm{D}$-Crystallin. Protein Sci. 2005, 14, 569-581.

(24) Serebryany, E.; King, J. A. The $\beta \gamma$-crystallins: Native state stability and pathways to aggregation. Prog. Biophys. Mol. Biol. 2014, $115,32-41$.

(25) Sahin, E.; Jordan, J. L.; Spatara, M. L.; Naranjo, A.; Costanzo, J. A.; Weiss, W. F.; Robinson, A. S.; Fernandez, E. J.; Roberts, C. J. Computational design and biophysical characterization of aggregation-resistant point mutations for $\gamma \mathrm{D}$ Crystallin illustrate a balance of conformational stability and intrinsic aggregation propensity. Biochemistry 2011, 50, 628-639.

(26) Flaugh, S. L.; Mills, I. A.; King, J. Glutamine deamidation destabilizes human $\gamma \mathrm{D}$-Crystallin and lowers the kinetic barrier to unfolding. J. Biol. Chem. 2006, 281, 30782-30793.

(27) Mills, I. A.; Flaugh, S. L.; Kosinski-Collins, M. S.; King, J. A. Folding and stability of the isolated Greek key domains of the longlived human lens proteins $\gamma \mathrm{D}$ Crystallin and $\gamma \mathrm{S}$ Crystallin. Protein Sci. 2007, 16, 2427-2444.

(28) Bennett, M.; Schlunegger, M.; Eisenberg, D. 3D domain swapping - a mechanism for oligomer assembly. Protein Sci. 1995, 4, $2455-2468$.

(29) Liu, Y.; Eisenberg, D. 3D domain swapping: as domains continue to swap. Protein Sci. 2002, 11, 1285-1299.

(30) Mascarenhas, N. M.; Gosavi, S. Protein domain-swapping can be a consequence of functional residues. J. Phys. Chem. B 2016, 120, 6929-6938.

(31) Mascarenhas, N. M.; Gosavi, S. Understanding protein domainswapping using structure-based models of protein folding. Prog. Biophys. Mol. Biol. 2017, 128, 113-120.

(32) Yang, S.; Cho, S.; Levy, Y.; Cheung, M.; Levine, H.; Wolynes, P.; Onuchic, J. Domain swapping is a consequence of minimal frustration. Proc. Natl. Acad. Sci. U. S. A. 2004, 101, 13786-13791.

(33) van der Wel, P. C. A. Domain swapping and amyloid fibril conformation. Prion 2012, 6, 211-216.

(34) Knaus, K.; Morillas, M.; Swietnicki, W.; Malone, M.; Surewicz, W.; Yee, V. Crystal structure of the human prion protein reveals a mechanism for oligomerization. Nat. Struct. Biol. 2001, 8, 770-774.

(35) Janowski, R.; Kozak, M.; Abrahamson, M.; Grubb, A.; Jaskolski, M. 3D domain-swapped human Cystatin $\mathrm{C}$ with amyloidlike intermolecular $\beta$-sheets. Proteins: Struct., Funct., Genet. 2005, 61, $570-578$.

(36) Nilsson, M.; Wang, X.; Rodziewicz-Motowidlo, S.; Janowski, R.; Lindstrom, V.; Onnerfjord, P.; Westermark, G.; Grzonka, Z.; Jaskolski, M.; Grubb, A. Prevention of domain swapping inhibits dimerization and amyloid fibril formation of cystatin C - use of engineered disulfide bridges, antibodies, and carboxymethylpapain to stabilize the monomeric form of cystatin C. J. Biol. Chem. 2004, 279, 24236-24245.

(37) Bacarizo, J.; Martinez-Rodriguez, S.; Martin-Garcia, J. M.; Andujar-Sanchez, M.; Ortiz-Salmeron, E.; Neira, J. L.; Camara- 
Artigas, A. Electrostatic effects in the folding of the SH3 domain of the c-Src Tyrosine Kinase: $\mathrm{pH}$-dependence in 3D-domain swapping and amyloid formation. PLoS One 2014, 9, e113224.

(38) Liu, C.; Sawaya, M. R.; Eisenberg, D. $\beta 2$-microglobulin forms three-dimensional domain-swapped amyloid fibrils with disulfide linkages. Nat. Struct. Mol. Biol. 2011, 18, 49-55.

(39) Pica, A.; Merlino, A.; Buell, A. K.; Knowles, T. P. J.; Pizzo, E.; D’Alessio, G.; Sica, F.; Mazzarella, L. Three-dimensional domain swapping and supramolecular protein assembly: insights from the $\mathrm{X}$ ray structure of a dimeric swapped variant of human pancreatic RNase. Acta Crystallogr., Sect. D: Biol. Crystallogr. 2013, 69, 21162123.

(40) Ercole, C.; Lopez-Alonso, J. P.; Font, J.; Ribo, M.; Vilanova, M.; Picone, D.; Laurents, D. V. Crowding agents and osmolytes provide insight into the formation and dissociation of RNase A oligomers. Arch. Biochem. Biophys. 2011, 506, 123-129.

(41) Liu, Y.; Gotte, G.; Libonati, M.; Eisenberg, D. A domainswapped RNase A dimer with implications for amyloid formation. Nat. Struct. Biol. 2001, 8, 211-214.

(42) Rousseau, F.; Schymkowitz, J.; Wilkinson, H.; Itzhaki, L. Threedimensional domain swapping in p13sucl occurs in the unfolded state and is controlled by conserved proline residues. Proc. Natl. Acad. Sci. U. S. A. 2001, 98, 5596-5601.

(43) Serebryany, E.; Woodard, J. C.; Adkar, B. V.; Shabab, M.; King, J. A.; Shakhnovich, E. I. An internal disulfide locks a misfolded aggregation-prone intermediate in cataract-linked mutants of human $\gamma$ D-Crystallin. J. Biol. Chem. 2016, 291, 19172-19183.

(44) Acosta-Sampson, L.; King, J. Partially folded aggregation intermediates of human $\gamma \mathrm{D}$-, $\gamma \mathrm{C}$-, and $\gamma \mathrm{S}$-Crystallin are recognized and bound by human $\alpha \mathrm{B}$-Crystallin chaperone. J. Mol. Biol. 2010, 401, 134-152.

(45) Dixit, K.; Pande, A.; Pande, J.; Sarma, S. P. Nuclear magnetic resonance structure of a major lens protein, human $\gamma \mathrm{C}$-Crystallin: role of the dipole moment in protein solubility. Biochemistry 2016, 55, 3136-3149.

(46) Basak, A.; Bateman, O.; Slingsby, C.; Pande, A.; Asherie, N.; Ogun, O.; Benedek, G.; Pande, J. High-resolution X-ray crystal structures of human $\gamma \mathrm{D}$ Crystallin $(1.25 \AA)$ and the $\mathrm{R} 58 \mathrm{H}$ mutant (1.15 ̊) associated with aculeiform cataract. J. Mol. Biol. 2003, 328, $1137-1147$.

(47) Hyeon, C.; Dima, R. I.; Thirumalai, D. Pathways and kinetic barriers in mechanical unfolding and refolding of RNA and proteins. Structure 2006, 14, 1633-1645.

(48) Liu, Z.; Reddy, G.; O’Brien, E. P.; Thirumalai, D. Collapse kinetics and chevron plots from simulations of denaturant-dependent folding of globular proteins. Proc. Natl. Acad. Sci. U. S. A. 2011, 108, 7787-7792.

(49) Bax, B.; Lapatto, R.; Nalini, V.; Driessen, H.; Lindley, P.; Mahadevan, D.; Blundell, T.; Slingsby, C. X-ray-analysis of $\beta \mathrm{B} 2$ Crystallin and evolution of oligomeric lens proteins. Nature 1990, 347, 776-780.

(50) Lapatto, R.; Nalini, V.; Bax, B.; Driessen, H.; Lindley, P.; Blundell, T.; Slingsby, C. High-resolution structure of an oligomeric eye lens $\beta$-Crystallin - loops, arches, linkers and interfaces in $\beta \mathrm{B} 2$ dimer compared to a monomeric $\gamma$-Crystallin. J. Mol. Biol. 1991, 222, $1067-1083$.

(51) Smith, M. A.; Bateman, O. A.; Jaenicke, R.; Slingsby, C. Mutation of interfaces in domain-swapped human $\beta \mathrm{B} 2$-Crystallin. Protein Sci. 2007, 16, 615-625.

(52) Jung, J.; Byeon, I. J. L.; Wang, Y.; King, J.; Gronenborn, A. M. The structure of the cataract-causing P23T mutant of human $\gamma \mathrm{D}$ Crystallin exhibits distinctive local conformational and dynamic changes. Biochemistry 2009, 48, 2597-2609.

(53) Pande, A.; Annunziata, O.; Asherie, N.; Ogun, O.; Benedek, G.; Pande, J. Decrease in protein solubility and cataract formation caused by the Pro23 to Thr mutation in human $\gamma \mathrm{D}$-Crystallin. Biochemistry 2005, 44, 2491-2500.

(54) Pande, A.; Pande, J.; Asherie, N.; Lomakin, A.; Ogun, O.; King, J.; Lubsen, N.; Walton, D.; Benedek, G. Molecular basis of a progressive juvenile-onset hereditary cataract. Proc. Natl. Acad. Sci. U. S. A. 2000, 97, 1993-1998.

(55) Santhiya, S.; Manohar, M.; Rawlley, D.; Vijayalakshmi, P.; Namperumalsamy, P.; Gopinath, P.; Loster, J.; Graw, J. Novel mutations in the $\gamma$-Crystallin genes cause autosomal dominant congenital cataracts. J. Med. Genet. 2002, 39, 352-358.

(56) Bennett, M. J.; Sawaya, M. R.; Eisenberg, D. Deposition diseases and 3D domain swapping. Structure 2006, 14, 811-824.

(57) Betancourt, M.; Thirumalai, D. Pair potentials for protein folding: choice of reference states and sensitivity of predicted native states to variations in the interaction schemes. Protein Sci. 1999, 8, $361-369$.

(58) Ding, F.; Dokholyan, N.; Buldyrev, S.; Stanley, H.; Shakhnovich, E. Molecular dynamics simulation of the SH3 domain aggregation suggests a generic amyloidogenesis mechanism. J. Mol. Biol. 2002, 324, 851-857.

(59) Ermak, D.; Mccammon, J. Brownian dynamics with hydrodynamic interactions. J. Chem. Phys. 1978, 69, 1352-1360.

(60) Li, X. Q.; Cai, H. C.; Zhou, S. Y.; Yang, J. H.; Xi, Y. B.; Gao, X. B.; Zhao, W. J.; Li, P.; Zhao, G. Y.; Tong, Y.; et al. A novel mutation impairing the tertiary structure and stability of $\gamma \mathrm{C}$-Crystallin (CRYGC) leads to cataract formation in humans and zebrafish lens. Hum. Mutat. 2012, 33, 391-401.

(61) Kumar, S.; Rosenberg, J. M.; Bouzida, D.; Swendsen, R. H.; Kollman, P. A. The weighted histogam analysis method for freeenergy calculations on biomolecules. J. Comput. Chem. 1992, 13, $1011-1021$.

(62) Wong, E. K.; Prytkova, V.; Freites, J. A.; Butts, C. T.; Tobias, D. J. Molecular mechanism of aggregation of the cataract-related $\gamma \mathrm{D}$ Crystallin W42R variant from multiscale atomistic simulations. Biochemistry 2019, 58, 3691-3699.

(63) Pande, A.; Ghosh, K. S.; Banerjee, P. R.; Pande, J. Increase in surface hydrophobicity of the cataract-associated P23T mutant of human $\gamma \mathrm{D}$-Crystallin is responsible for its dramatically lower, retrograde solubility. Biochemistry 2010, 49, 6122-6129.

(64) Pande, A.; Zhang, J.; Banerjee, P. R.; Puttamadappa, S. S.; Shekhtman, A.; Pande, J. NMR study of the cataract-linked P23T mutant of human $\gamma \mathrm{D}$-Crystallin shows minor changes in hydrophobic patches that reflect its retrograde solubility. Biochem. Biophys. Res. Commun. 2009, 382, 196-199.

(65) Hanson, S.; Hasan, A.; Smith, D.; Smith, J. The major in vivo modifications of the human water-insoluble lens crystallins are disulfide bonds, deamidation, methionine oxidation and backbone cleavage. Exp. Eye Res. 2000, 71, 195-207.

(66) Boatz, J. C.; Whitley, M. J.; Li, M.; Gronenborn, A. M.; van der Wel, P. C. A. Cataract-associated P23T $\gamma$ D-Crystallin retains a nativelike fold in amorphous-looking aggregates formed at physiological $\mathrm{pH}$. Nat. Commun. 2017, 8, 15137.

(67) Serebryany, E.; Yu, S.; Trauger, S. A.; Budnik, B.; Shakhnovich, E. I. Dynamic disulfide exchange in a Crystallin protein in the human eye lens promotes cataract-associated aggregation. J. Biol. Chem. 2018, 293, 17997-18009.

(68) Reddy, G.; Liu, Z.; Thirumalai, D. Denaturant-dependent folding of GFP. Proc. Natl. Acad. Sci. U. S. A. 2012, 109, 1783217838 .

(69) Reddy, G.; Thirumalai, D. Dissecting ubiquitin folding using the self-organized polymer model. J. Phys. Chem. B 2015, 119, 11358-11370.

(70) Zhuravlev, P. I.; Reddy, G.; Straub, J. E.; Thirumalai, D. Propensity to form amyloid fibrils is encoded as excitations in the free energy landscape of monomeric proteins. J. Mol. Biol. 2014, 426, $2653-2666$

(71) Mondal, B.; Reddy, G. Cosolvent effects on the growth of protein aggregates formed by a single domain globular protein and an intrinsically disordered protein. J. Phys. Chem. B 2019, 123, 19501960.

(72) Mondal, B.; Reddy, G. A transient intermediate populated in prion folding leads to domain swapping. Biochemistry 2020, 59, 114124. 
(73) Giblin, F. Glutathione: a vital lens antioxidant. J. Ocul. Pharmacol. Ther. 2000, 16, 121-135.

(74) Berthoud, V. M.; Beyer, E. C. Oxidative stress, lens gap junctions, and cataracts. Antioxid. Redox Signaling 2009, 11, 339-353.

(75) Hains, P. G.; Truscott, R. J. W. Post-translational modifications in the nuclear region of young, aged, and cataract human lenses. $J$. Proteome Res. 2007, 6, 3935-3943.

(76) Michael, R.; Bron, A. J. The ageing lens and cataract: a model of normal and pathological ageing. Philos. Trans. R. Soc., B 2011, 366, $1278-1292$.

(77) Hains, P. G.; Truscott, R. J. W. Proteomic analysis of the oxidation of cysteine residues in human age-related nuclear cataract lenses. Biochim. Biophys. Acta, Proteins Proteomics 2008, 1784, 19591964.

(78) Spector, A.; Roy, D. Disulfide-linked high molecular-weight protein associated with human cataract. Proc. Natl. Acad. Sci. U. S. A. 1978, 75, 3244-3248.

(79) Fan, X.; Zhou, S.; Wang, B.; Hom, G.; Guo, M.; Li, B.; Yang, J.; Vaysburg, D.; Monnier, V. M. Evidence of highly conserved $\beta$ Crystallin disulfidome that can be mimicked by in vitro oxidation in age-related human cataract and glutathione depleted mouse lens. Mol. Cell. Proteomics 2015, 14, 3211-3223.

(80) Francis Simpanya, M.; Ansari, R. R.; Leverenz, V.; Giblin, F. J. Measurement of lens protein aggregation in vivo using dynamic light scattering in a guinea pig/uva model for nuclear cataract. Photochem. Photobiol. 2008, 84, 1589-1595.

(81) Thorn, D. C.; Grosas, A. B.; Mabbitt, P. D.; Ray, N. J.; Jackson, C. J.; Carver, J. A. The structure and stability of the disulfide-linked $\gamma \mathrm{S}$-Crystallin dimer provide insight into oxidation products associated with lens cataract formation. J. Mol. Biol. 2019, 431, 483-497.

(82) Muttathukattil, A. N.; Singh, P. C.; Reddy, G. Role of disulfide bonds and topological frustration in the kinetic partitioning of lysozyme folding pathways. J. Phys. Chem. B 2019, 123, 3232-3241.

(83) Qin, M.; Wang, W.; Thirumalai, D. Protein folding guides disulfide bond formation. Proc. Natl. Acad. Sci. U. S. A. 2015, 112, 11241-11246. 